\title{
Effect of Non-Surgical Periodontal Therapy on the Severity of Rheumatoid Arthritis-A Clinical and Hematological Study
}

\author{
Rujuta Krishnakant Pandya1, Monali Amit Shah², Yesha Hareshkumar Raval ${ }^{3}$ \\ ${ }^{1}$ K. M. Shah Dental College and Hospital, Sumandeep Vidyapeeth, Rajkot, India \\ ${ }^{2}$ Department of Periodontology, K. M. Shah Dental College and Hospital, Sumandeep Vidyapeeth, Piparia, India \\ ${ }^{3}$ K. M. Shah Dental College and Hospital, Sumandeep Vidyapeeth, Surat, India \\ Email:docrujuta@gmail.com, smonali2011@gmail.com,yesha_raval@yahoo.in
}

How to cite this paper: Pandya, R.K., Shah, M.A. and Raval, Y.H. (2020) Effect of Non-Surgical Periodontal Therapy on the Severity of Rheumatoid Arthritis-A Clinical and Hematological Study. Open Journal of Stomatology, 10, 1-9.

https://doi.org/10.4236/ojst.2020.101001

Received: November 13, 2019

Accepted: December 30, 2019

Published: January 2, 2020

Copyright $\odot 2020$ by author(s) and Scientific Research Publishing Inc. This work is licensed under the Creative Commons Attribution International License (CC BY 4.0).

http://creativecommons.org/licenses/by/4.0/

\begin{abstract}
Background: The epidemiological and pathological relations between Periodontitis and Rheumatoid arthritis (RA) have been stated. The citrullination and attendant autoantibody responses are defining features of RA. It has been recognized that the oral bacteria, inflammation and its mediators might play an important role in RA activity. Hence this study aimed to evaluate the effect of Non-surgical Periodontal Therapy in reducing the severity of Rheumatoid arthritis. Materials and Method: Totally 25 participants diagnosed with RA and Periodontitis were included in the study. Clinical parameters such as CPSS index, BOP\% and CAL, estimation of ESR and calculation of DAS28 done at baseline, after Pre-NSPT and after next Post-NSPT were recorded. Results: Statistically significant improvement was noted for CPSS ( $\mathrm{p}<$ $0.001)$, BOP\% $(\mathrm{p}<0.001)$ and CAL $(\mathrm{p}<0.001)$ after NSPT. The ESR values also showed significant reductions $(\mathrm{p}<0.001)$ and the DAS28 scores also showed statistically significant improvements $(\mathrm{p}<0.001)$ after NSPT. Conclusion: Reduction in the periodontal inflammation positively affects the rheumatoid arthritis activity, hence improving the patient's health.
\end{abstract}

\section{Keywords}

Chronic Periodontitis, Rheumatoid Arthritis, DAS28

\section{Introduction}

Periodontal diseases range widely from gingivitis to aggressive forms of periodontitis and along with being a threat to the dentition, they may also affect the systemic health [1]. 
Periodontitis is a diseased state that is portrayed as an inflammatory state and bacterial disease driven by various factors. Gingivitis is inflammation caused by formation of dental plaque and its elimination is possible by reducing the bacterial load through oral hygiene measures. But, when the infection spreads, destructive periodontal disease develops. Subgingival plaque is composed of various bacterial species consisting mostly of strict anaerobes. A significant part of these is non-cultivable including Aggregatibacter actinomycetemcomitans (A.a), Porphyromonas gingivalis (P.g), Prevotella intermedia (P.i), Parvimonas micra, Tannerella forsythia and treponema species. Periodontitis leads to break-down of periodontium. Clinically, this condition would be evident as bleeding on probing, deepened periodontal pockets, suppuration, mobility and eventually tooth and bone loss [2].

The continuous signs of inflammation from the body may also be caused due to severe periodontitis. An association with chronic and inflammatory systemic diseases such as diabetes mellitus, artherosclerosis, cardiovascular disease and stroke, rheumatoid arthritis (RA), Crohn's disease, ulcerative colitis, low birth weight \& preterm birth has also been noted [2].

RA is a common disease which is of autoimmune origin \& progressively disables the individual with systemic complications \& also leads to early death and socioeconomic costs. It's an inflammatory destructive disease which is chronic in nature and characterized by inflammation and hyperplasia of synovial joints, autoantibody production (anti-citrullinated protein antibody [ACPA] \& rheumatoid factor $[R F]$ ), deformity of cartilage \& bone and systemic features which include cardiovascular, pulmonary, psychological and skeletal disorders. An accumulation of cells responsible for inflammation including $\mathrm{T}$ and $\mathrm{B}$ cells lymphocytes, monocytes and neutrophils is seen [1] [3]. Long lasting synovial inflammation that causes damage to articular cartilage and the underlying bone is due to several inflammatory cascades which lead to immune responses ultimately leading to common pathway [2]. RA being a systemic disease, has some extra-articular manifestations like pulmonary, ocular, vascular and other organs or that may be affected by inflammatory process [1].

RA has exhibited similarities with periodontitis in terms of inflammation and destruction of tissues. These include: chronic destructive inflammatory disorders with a characteristic of deregulating the host inflammatory response, they have a multifactorial etiology, shared genetic and the lifestyle factors influence the disease susceptibility and the longer duration of disease decreases the severity, loss of function \& quality of life [2]. This indicates sufficient similarities of the underlying pathologies [4]. The microorganisms which are responsible for RA and the periodontal pathogens share many features. Shah, M. et al. in 2013 showed improvement in pregnancy outcomes in terms of pre-term low birth weight after the treatment of $\mathrm{CP}$ during gestation. This would suggest a correlation [5].

The severity of a disease may be caused due to amount and type of cytokines which the individual will produce. For certain populations a correlation with 
polymorphisms in interleukin-1 (IL-1) gene cluster has been seen for periodontitis.

A significant reduction in ESR (Erythrocyte Sedimentation Rate) with periodontal treatment was seen by Al-Katma, M.K. et al. [4]. Even with the RA medications like NSAIDs and immunosuppressants which can reduce periodontal destruction, significant destruction has been noted [1].

The studies up to date show conflicting results. In last decade, the interest in epidemiological and pathological connections between CP and RA has shown a rise which may be caused by role of citrullination and the attendant autoantibody responses which would be the disease-defining feature for RA and may be recognized that oral bacterial inflammation may play important roles.

\section{Materials and Method}

This was a non-randomized cross over, clinical and hematological study. The patients were selected from the OPD of the Department of Periodontology, K. M. Shah Dental College and Hospital, as well as from the Department of General Medicine and Department of Orthopaedics, Dhiraj General Hospital, Pipariya, Vadodara.

Considering the difference in group means to be $20 \%$, power of the study as $80 \%$, at $95 \%$ confidence interval, a ratio of sample size (group 1/group 2) as 1 and with the significance level set at $5 \%$, a sample size of 25 in each group was taken.

Any participant who had been diagnosed by a physician, as having active rheumatoid arthritis, i.e., the destructive inflammatory condition is ongoing, since 1 year, had been selected for the study. Such patients, when examined clinically, if diagnosed as having chronic periodontitis, i.e., having inflamed gingiva with bleeding on probing and pocket probing depth $\geq 5 \mathrm{~mm}$ where selected for the study.

Participants between the age range of $30-70$ years with at least 20 natural teeth and those who had not received any periodontal therapy in the past 6 months were included. Those having any systemic disease or immune anomalies other than RA were excluded. Also, pregnant and lactating females and/or those on hormone replacement therapy and oral contraceptives \& smokers and/or alcoholics were excluded.

Details regarding the study and their role for the same were explained to each participant and an informed consent was signed by them. On the first appointment, complete medical and dental histories were taken. Oral examination was done and the clinical parameters were recorded using an UNC-15 probe, which included: CPSS index (Clinical Periodontal Sum Score), BOP\% (Bleeding on Probing \%) and CAL (Clinical Attachment Loss). ESR (Erythrocyte Sedimentation Rate) was tested. Also, DAS28 (Disease Activity Score) was recorded where the number of tender and swollen joints were marked. Oral hygiene instructions were given and the participants were kept on follow up. 
In the second appointment, all the clinical parameters along with ESR and DAS28 were recorded again. Then after the participants were treated with non-surgical periodontal therapy (NSPT) which included scaling and root planing, oral hygiene instructions were given and the participants were recalled.

In the third appointment, the clinical parameters were recorded along with ESR and DAS28.

\subsection{Estimation of ESR (Erythrocyte Sedimentation Rate)}

With patient's consent, prior to each sample collection, disinfection of the skin at the site of venepuncture will be done with surgical spirit.

After tying the tourniquet 3 - 4 inches above the antecubital fossa, blood sample of $3 \mathrm{ml}$ will be collected from the antecubital vein of the forearm using 20 gauge needle and $5 \mathrm{ml}$ syringe and stored in tubes containing tri-sodium citrate anticoagulant.

The anticoagulated blood would be placed in an upright tube, known as a Westergren tube, and the rate at which the red blood cells fall would be measured and reported in $\mathrm{mm} / \mathrm{h}$.

\subsection{DAS28 (Disease Activity Score 28)}

DAS28 was used as it combines single measures into an overall continuous measure of rheumatoid arthritis disease activity and this index has an advantage of simultaneous interpretation of several measures of RA like swollen and tender joint count, erythrocyte sedimentation rate and general health assessment on a visual analogue scale [6].

For recording DAS28, an online calculator was used where the number of tender and swollen joints was marked and ESR was entered. The calculation was done using the formulae:

$$
\operatorname{DAS} 28(4)=* \operatorname{sqrt}(\operatorname{sw} 28)+0.70 * \operatorname{Ln}(\mathrm{ESR})+0.014 * \mathrm{GH}
$$

\subsection{CPSS (Clinical Periodontal Sum Score)}

CPSS index was used to provide one representative value per individual to escribe the periodontal status. As suggested by Matilla, K.J. et al. [7], the following parameters were included.

PPD $4 \mathrm{~mm}$ or greater + gingival sites with $\mathrm{BOP}+$ visible suppuration on probing + furcation lesions exceeding grade 1 .

\section{Results}

Total 25 participants with RA and chronic periodontitis were included. Participants crossed over from control arm to test arm accounting to 50 sample size in total. The mean age of the participants was $48.24 \pm 9.02$ years including $36 \%$ male and $64 \%$ female. Clinical Periodontal Sum Score at baseline was $104.32 \pm$ 39.862 , at pre-NSPT was $105 \pm 39.976$ and at post-NSPT was $14.28 \pm 8.284$. This shows a statistical significant improvement in the score with $\mathrm{p}$ value $<0.001$ 
(Table 1). Bleeding on probing percentage at baseline was $85.81 \pm 15.366$, at pre-NSPT was $85.96 \pm 15.489$ and at post-NSPT was $17.96 \pm 10.725$. This shows a statistical significant improvement in the score with $\mathrm{p}$ value $<0.001$ (Table 1 ). Clinical Attachment level at baseline was $5.13 \pm 1.496$, at pre-NSPT was $5.16 \pm$ 1.493 and at post-NSPT was $3.95 \pm 1.044$.

This shows a statistical significant improvement in the score with $\mathrm{p}$ value < 0.001 (Table 1). Erythrocyte Sedimentation Rate (ESR) at baseline was $55.04 \pm$ 8.233 , at pre-NSPT was $55.04 \pm 8.233$ and at post-NSPT was $23.92 \pm 6.082$. This shows a statistical significant improvement in the score with $\mathrm{p}$ value $<0.001$ (Table 2). Disease Activity Score at 28 joints (DAS28) for Rheumatoid Arthritis was calculated at baseline was $5.259 \pm 0.788$, at pre-NSPT was $5.259 \pm 0.788$ and at post-NSPT was $4.492 \pm 0.760$. This shows a statistical significant improvement in the score with $\mathrm{p}$ value $<0.001$ (Table 2 ).

\section{Discussion}

The main aim of this study was to evaluate the effect of NAPT on the disease severity of rheumatoid arthritis.

RA includes an initiating event which is possibly a microbial exposure or a putative autoantigen which leads to significant synovial inflammation and tissue destruction. Periodontitis also includes the inflammatory cell accumulation which includes $\mathrm{T}$ and $\mathrm{B}$ lymphocytes, neutrophils and monocytes. This leads to tissue edema, endothelial cell proliferation and matrix degradation [1].

In cases of active rheumatoid arthritis, it has been estimated that more than a billion neutrophils actively participate in the immune defence and lead to an active inflammatory burden on the systemic health. This burden would reduce in cases where the disease is not actively destructive. Hence participants with active rheumatoid arthritis with ages 30 - 70 years were selected.

Similarly, participants treated for periodontal disease in the past 6 months would not show an active periodontal destruction and hence would not be associated with the inflammatory condition. Hence participants who had undergone

Table 1. Comparison of periodontal parameters before and after treatment.

\begin{tabular}{ccccc}
\hline Parameters & Baseline & Pre NSPT & Post NSPT & p Value $^{*}$ \\
\hline CAL & $5.13 \pm 1.49$ & $5.16 \pm 1.49$ & $3.95 \pm 1.04$ & $<0.001$ \\
BOP\% & $85.81 \pm 15.36$ & $85.96 \pm 15.48$ & $17.96 \pm 10.72$ & $<0.001$ \\
CPSS & $104.32 \pm 39.86$ & $105.00 \pm 39.97$ & $14.28 \pm 8.28$ & $<0.001$ \\
\hline
\end{tabular}

*Using ANOVA and Wilk's lambda test.

Table 2. Comparison of systemic parameters before and after treatment.

\begin{tabular}{ccccc}
\hline Parameters & Baseline & Pre NSPT & Post NSPT & p Value $^{*}$ \\
\hline Das28 & $5.25 \pm 0.78$ & $5.25 \pm 0.78$ & $4.49 \pm 0.76$ & $<0.001$ \\
ESR & $55.04 \pm 8.23$ & $55.04 \pm 8.23$ & $23.92 \pm 6.08$ & $<0.001$ \\
\hline
\end{tabular}

*Using ANOVA and Wilk's lambda test. 
any periodontal treatment in previous 6 months were not included in the study.

Participants with any other systemic condition of diseases that would be responsible for the systemic inflammatory burden, destructive reaction of hormonal changes that might be responsible for these were excluded from the study.

Observations from natural history studies of RA and periodontitis have suggested that such populations are characterized by a particular type of patient who will experience disease progression irrespective of any treatment given [1]. Pablo et al. in 2008 stated that periodontitis is twice as common and severe in patients with RA and such patients were twice as likely to become edentulous [8]. P. gingivalis, the periodontal pathogen invades the human endothelium cells by impairing the epithelial integrity and influences the transcription and protein synthesis [9]. Hence, these organisms have a direct systemic access to the blood circulation [10]. It has shown to invade the primary human chondrocytes isolated from knee joints and delay the cell cycle progression and increased cell apoptosis in these chondrocytes [11].

The pathogenesis of periodontitis and RA includes the upregulation of variety of cytokines and matrix metalloproteinases (MMPs). The cytokine profiles that are similar in both these diseases consist of persistent high levels of proinflammatory cytokines including IL- $1 \beta$ and tumor necrosis factor-alpha (TNF- $\alpha$ ) and low levels of cytokines that suppress the immunoinflammatory response like IL-10 and transforming growth factor- $\beta$ (TGF- $\beta$ ). These cytokines along with low levels of tissue inhibitors of metalloproteinases (TIMPs) and high levels of MMPs and prostaglandin $\mathrm{E}_{2}\left(\mathrm{PGE}_{2}\right)$ also has association with active periodontitis. For the regulation of osteoclast formation and activation, the RANKL and its receptor and cell surface TNF-like molecule are the key factors [1].

In this study there was statistically significant improvement in all the periodontal parameters including bleeding on probing and clinical attachment level after the non-surgical periodontal therapy which re-establishes the fact that dental deposits like plaque and calculus are the primary etiologic factor for periodontal disease and that their elimination would improve the periodontal health.

The study showed a highly statistical significant decrease in the ESR levels between baseline and post-NSPT. A systematic review conducted by Chambrone L. et al. in 2013 included five studies and concluded that non-surgical periodontal therapy was associated with decreased ESR and a trend to decreased TNF and DAS28 score [12].

The study showed a highly statistical significant decrease in the disease activity of rheumatoid arthritis following the non-surgical periodontal therapy. The decrease in DAS28 score was most likely the result of the non-surgical periodontal therapy, as the participants were checked for other risk factors including drug history and other possibly related systemic conditions [13]. Kaur, S. et al. in 2014 concluded that non-surgical periodontal treatment improved the markers of RA disease activity [14].

William and Offenbacher stated that with the severity of the periodontal pock- 
ets, the number of plasma cells also increased. Hence it can be concluded that the periodontal inflammation stimulates the production of globulins, C-reactive proteins, fibrinogens and plasma proteins. The decrease in the severity of RA in this study would be due to the decrease in the inflammatory products in the blood, following the non-surgical periodontal therapy. D'Aiuto et al. showed a significant reduction in IL-6 and CRP two months following non-surgical periodontal therapy [15]. The reduced exposure of the joint structures to bacteria and their products by elimination of bacterial plaque and endotoxins may contribute in improvement of RA condition [16].

Vice-versa, Kaber et al. in 1997 stated that patients with longstanding active RA have a substantially increased frequency of periodontal disease, including loss of teeth and that anti-inflammatory treatment interferes with periodontal disease [17]. Mayer et al. in 2009 showed that RA patients undergoing anti-TNF-x medication had lower periodontal indices and GCF TNF-x levels which might mean that the suppression of proinflammatory cytokines might prove beneficial in reduction of periodontal disease [18].

Contradictory to these observations, a study done by Mercado e al in 2001 concluded that no differences were noted for the plaque and bleeding indices between the healthy and rheumatoid arthritis patients [19].

The limitations of this study include the smaller sample size and shorter follow up period.

\section{Conclusion}

Comparing the reduced values of periodontal status and the rheumatoid condition correlated to a connection between the conditions, which would be attributed to the reduction of the inflammatory burden from the system, this may conclude that periodontitis and RA have various pathologies in common and that further studies with larger sample sizes and improved study designs should be carried to establish a correlation.

\section{Future Implications}

- Studies involving RCT.

- More studies with longer period of follow-ups are needed.

- Multi-center studies with larger sample size.

\section{Financial Support and Sponsorship}

All the expenditure was borne by the primary investigator.

\section{Conflicts of Interest}

There are no conflicts of interest.

\section{References}

[1] Bartold, P.M., Marshall, R.I. and Haynes, D.R. (2005) Periodontitis and Rheumatoid 
Arthritis: A Review. Journal of Periodontology, 76, 2066-2074. https://doi.org/10.1902/jop.2005.76.11-S.2066

[2] De Smit, M.J., Brouwer, E., Vissink, A. and Van Winkelhoff, A.J. (2011) Rheumatoid Arthritis and Periodontitis; a Possible Link via Citrullination. Anaerobe, 17, 196-200. https://doi.org/10.1016/j.anaerobe.2011.03.019

[3] McInnes, I.B. and Schett, G. (2011) The Pathogenesis of Rheumatoid Arthritis. New England Journal of Medicine, 365, 2205-2219. https://doi.org/10.1056/NEJMra1004965

[4] Al-Katma, M.K., Bissada, N.F., Bordeaux, J.M., Sue, J. and Askari, A.D. (2007) Control of Periodontal Infection Reduces the Severity of Active Rheumatoid Arthritis. Journal of Clinical Rheumatology, 13, 134-137. https://doi.org/10.1097/RHU.0b013e3180690616

[5] Shah, M., Muley, A. and Muley, P. (2013) Effect of Nonsurgical Periodontal Therapy during Gestation Period on Adverse Pregnancy Outcome: A Systematic Review. The Journal of Maternal-Fetal \& Neonatal Medicine, 26, 1691-1695. https://doi.org/10.3109/14767058.2013.799662

[6] Fransen, J., Stucki, G. and van Riel, P.L. (2003) Rheumatoid Arthritis Measures: Disease Activity Score (DAS), Disease Activity Score-28 (DAS28), Rapid Assessment of Disease Activity in Rheumatology (RADAR), and Rheumatoid Arthritis Disease Activity Index (RADAI). Arthritis Care \& Research: Official Journal of the American College of Rheumatology, 49, S214-S224.

[7] Mattila, K.J., Asikainen, S., Wolf, J., Jousimies-Somer, H., Valtonen, V. and Nieminen, M. (2000) Age, Dental Infections, and Coronary Heart Disease. Journal of Dental Research, 79, 756-760. https://doi.org/10.1177/00220345000790020901

[8] De Pablo, P., Dietrich, T. and McAlindon, T.E. (2008) Association of Periodontal Disease and Tooth Loss with Rheumatoid Arthritis in the US Population. The Journal of Rheumatology, 35, 70-76.

[9] Lundberg, K., Kinloch, A., Fisher, B.A., Wegner, N., Wait, R., Charles, P., Mikuls, T.R. and Venables, P.J. (2008) Antibodies to Citrullinated $\alpha$-Enolase Peptide 1 Are Specific for Rheumatoid Arthritis and Cross-React with Bacterial Enolase. Arthritis \& Rheumatology, 58, 3009-3019. https://doi.org/10.1002/art.23936

[10] Lu, J., Zhang, W., Hao, Y. and Zhu, Y. (2009) Defect of Cell Wall Construction May Shield Oral Bacteria's Survival in Bloodstream and Cause Infective Endocarditis. Medical Hypotheses, 73, 1055-1057. https://doi.org/10.1016/j.mehy.2009.05.018

[11] Pischon, N., Roehner, E., Hocke, A., N'Guessan, P., Mueller, H.C., Matziolis, G., Kanitz, V., Purucker, P., Kleber, B.M., Bernimoulin, J.P. and Burmester, G. (2009) Effects of Porphyromonas gingivalis on Cell Cycle Progression and Apoptosis of Primary Human Chondrocytes. Annals of the Rheumatic Diseases, 68, 1902-1907. https://doi.org/10.1136/ard.2008.102392

[12] Chambrone, L., Preshaw, P.M., Rosa, E.F., Heasman, P.A., Romito, G.A., Pannuti, C.M. and Tu, Y.K. (2013) Effects of Smoking Cessation on the Outcomes of Non-Surgical Periodontal Therapy: A Systematic Review and Individual Patient Data Meta-Analysis. Journal of Clinical Periodontology, 40, 607-615. https://doi.org/10.1111/jcpe.12106

[13] Williams, R.C. and Offenbacher, S. (2000) Periodontal Medicine: The Emergence of a New Branch of Periodontology. Periodontology, 23, 9-12. https://doi.org/10.1034/j.1600-0757.2000.2230101.x

[14] Kaur, S., Bright, R., Proudman, S.M. and Bartold, P.M. (2014) Does Periodontal Treatment Influence Clinical and Biochemical Measures for Rheumatoid Arthritis? 
A Systematic Review and Meta-Analysis. Seminars in Arthritis and Rheumatism, 44, 113-122. https://doi.org/10.1016/j.semarthrit.2014.04.009

[15] D'aiuto, F., Nibali, L., Parkar, M., Suvan, J. and Tonetti, M.S. (2005) Short-Term Effects of Intensive Periodontal Therapy on Serum Inflammatory Markers and Cholesterol. Journal of Dental Research, 84, 269-273.

https://doi.org/10.1177/154405910508400312

[16] Šimelyte, E., Rimpiläinen, M., Lehtonen, L., Zhang, X. and Toivanen, P. (2000) Bacterial Cell Wall-Induced Arthritis: Chemical Composition and Tissue Distribution of Four Lactobacillus Strains. Infection and Immunity, 68, 3535-3540. https://doi.org/10.1128/IAI.68.6.3535-3540.2000

[17] Käber, U.R., Michel, A., Bolten, W.W., Gleissner, C., Dehne, F. and Willershausen-Zönnchen, B. (1997) Risk for Periodontal Disease in Patients with Longstanding Rheumatoid Arthritis. Arthritis \& Rheumatism: Official Journal of the American College of Rheumatology, 40, 2248-2251.

https://doi.org/10.1002/art.1780401221

[18] Mayer, Y., Balbir-Gurman, A. and Machtei, E.E. (2009) Anti-Tumor Necrosis Factor-Alpha Therapy and Periodontal Parameters in Patients with Rheumatoid Arthritis. Journal of Periodontology, 80, 1414-1420.

https://doi.org/10.1902/jop.2009.090015

[19] Mercado, F.B., Marshall, R.I., Klestov, A.C. and Bartold, P.M. (2001) Relationship between Rheumatoid Arthritis and Periodontitis. Journal of Periodontology, 72, 779-787. https://doi.org/10.1902/jop.2001.72.6.779 